\title{
Carex divisa, una nueva ciperácea naturalizada en Chile
}

\author{
J. CALVO ${ }^{1} \&$ P. JIMÉNEZ-MEJÍAS ${ }^{2,3}$ \\ ${ }^{1}$ Instituto de Geografía, Facultad de Ciencias del Mar y Geografía, Pontificia Universidad Católica de Valparaíso, \\ av. Brasil, 2241, CL-2362807 Valparaíso, Chile \\ ${ }^{2}$ Departamento de Biología (Botánica), Universidad Autónoma de Madrid, Campus Cantoblanco, ES-28049 Madrid, \\ España \\ ${ }^{3}$ Centro de Investigación en Biodiversidad y Cambio Global (CIBC-UAM), Universidad Autónoma de Madrid, \\ ES-28049 Madrid, España \\ ORCID iD. J. CALVO: https://orcid.org/0000-0003-2340-7666, \\ P. JIMÉNEZ-MEJÍAS: https://orcid.org/0000-0003-2815-4477
}

Autor para correspondencia: P. Jiménez-Mejías (pjimmej@gmail.com)

Editores: T. Garnatje \& J. López-Pujol

Recibido 25 noviembre 2019; aceptado 1 abril 2020; publicado on line 1 julio 2020

\begin{abstract}
Carex divisa, a new Cyperaceae for the alien flora of Chile.- Carex divisa is recorded for the first time in Chile. A taxonomic discussion for its proper identification is provided.
\end{abstract}

Key words: alien species; Cyperaceae; Pacific coast; South America.

\section{Resumen}

Carex divisa, una nueva ciperácea naturalizada en Chile.-Carex divisa se registra por primera vez en Chile. Se incluye una discusión taxonómica para su apropiada determinación.

Palabras clave: costa del Pacífico; Cyperaceae; especies naturalizadas; Sudamérica.

\section{Cómo citar este artículo / Citation}

Calvo, J. \& Jiménez-Mejías, P. 2020. Carex divisa, una nueva ciperácea naturalizada en Chile. Collectanea Botanica 39: e008. https://doi.org/10.3989/collectbot.2020.v39.008

\section{Copyright}

(C) 2020 CSIC. This is an open-access article distributed under the terms of the Creative Commons Attribution 4.0 International (CC BY 4.0) License. 
El género Carex L. (Cyperaceae) es uno de los tres géneros de plantas vasculares más diversos del planeta, con cerca de 2000 especies y una distribución casi cosmopolita, aunque más diverso en las zonas templadas del hemisferio norte (Martín-Bravo et al., 2019). En Sudamérica el género comprende aproximadamente 200 especies, pertenecientes a todos sus grandes linajes excepto el llamado "clado Siderostictae" (Jiménez-Mejías et al., 2018a). Ello supone cerca del $10 \%$ de la diversidad mundial.

El conocimiento del género Carex en Sudamérica es fragmentario y aún requiere de mucho trabajo adicional. Desde la revisión general del género por Kükenthal (1909), los trabajos taxonómicos a lo largo de los dos primeros tercios del siglo XX fueron escasos, fragmentados y de ámbito localizado, con la excepción de las revisiones de Barros $(1935,1969)$ para el Cono Sur, Macbride (1936) para Perú y las contribuciones de Steyermark (1951) para Venezuela. No sería hasta el comienzo de la ingente labor de revisión de Gerald A. Wheeler cuando el género Carex (y su entonces género satélite Uncinia Pers.) ha comenzado a entenderse desde una perspectiva más integradora en Sudamérica (Wheeler, 1987, 1989, 1990, 2007, entre otros muchos trabajos). Recientemente, el segundo autor de la presente publicación tuvo la oportunidad de estudiar las mayores colecciones de Carex de Sudamérica en los Estados Unidos durante su etapa postdoctoral: $\mathrm{MICH}, \mathrm{MO}, \mathrm{NY}$ y US. Como resultado, distintos trabajos actualizando la taxonomía, nomenclatura y corología del género han sido publicados (p. ej. Jiménez-Mejías et al., 2016, 2018a, b; Jiménez-Mejías \& Silva, 2020).

En la presente nota damos conocimiento de un nuevo registro de Carex divisa Huds. en el continente sudamericano, la primera cita conocida para Chile. Esta especie pertenece al subgénero Vignea (P. Beauv. ex T. Lestib.) Peterm., que se caracteriza porque en la mayoría de las especies las espigas son bisexuales y sésiles (Egorova, 1999; Ball \& Reznicek, 2002). Carex divisa es una especie de distribución euroasiática, que se extiende desde la Península Ibérica hasta China occidental y que se conocía como introducida en Australia, Nueva Zelanda y Norteamérica (Govaerts et al., 2019). La presencia de C. divisa en Sudamérica ha sido reportada recientemente para Argentina y Uruguay (Jiménez-Mejías et al., 2018b). El taxon estaba erróneamente citado como C. marcida Boott (=C. praegracilis W. Boott) en la región del Río de la Plata (Herter, 1953; Myndel-Pedersen, 1968), con pliegos testigo que se remontan a 1937 (Jiménez-Mejías et al., 2018b). Carex divisa es fácilmente distinguible de cualquier otra especie de Carex en Sudamérica por la siguiente combinación de caracteres: rizomas reptantes, espigas sésiles andróginas y utrículos más largos que las glumas (Fig. 1A), con gruesos nervios prominentes que recorren la longitud completa del cuerpo del utrículo y con un pico netamente bífido (Fig. 1B). Se trata de una de las dos especies del género que parecen ser inequívocamente naturalizadas en el continente sudamericano, siendo la otra otro representante del subgénero Vignea, la también euroasiática C. divulsa Stokes (Barros, 1935; Myndel-Pedersen, 1968; Jiménez-Mejías et al., 2016).

La población encontrada en Chile (Fig. 1C) se localiza en la región de Valparaíso, específicamente al sur de la localidad costera de Quintay, una zona con cierta presión antrópica y esporádicamente frecuentada por ganado vacuno. Crece en la zona de transición entre la vegetación psammófila de los arenales costeros y una quebrada cubierta por matorral y bosque esclerófilo, siendo Pouteria splendens (A. DC.) Kuntze (Sapotaceae) una de las especies dominantes. La población ocupa unos $25 \mathrm{~m}^{2}$ y se desarrolla sobre un substrato arenoso y seco. Por lo que refiere a la fenología, los individuos se encontraron en floración en el mes de octubre y a mediados de noviembre la fructificación estaba terminando. Otra especie alóctona que se ha observado en la zona es Rosa rubiginosa L. Búsquedas adicionales en las proximidades de la zona (bien conocida por el primer autor de esta nota) no revelaron más núcleos poblacionales de $C$. divisa.

Estudios sobre el establecimiento de otras especies del género Carex en Sudamérica de forma natural muestran que éstas han expandido su nicho ecológico respecto a las poblaciones del hemisferio norte (Villaverde et al., 2017). Futuros trabajos enfocados a elucidar si dicho fenómeno tiene también lugar con especies introducidas por el hombre podría ayudar a cuantificar su potencial invasor y actuar en consecuencia. 


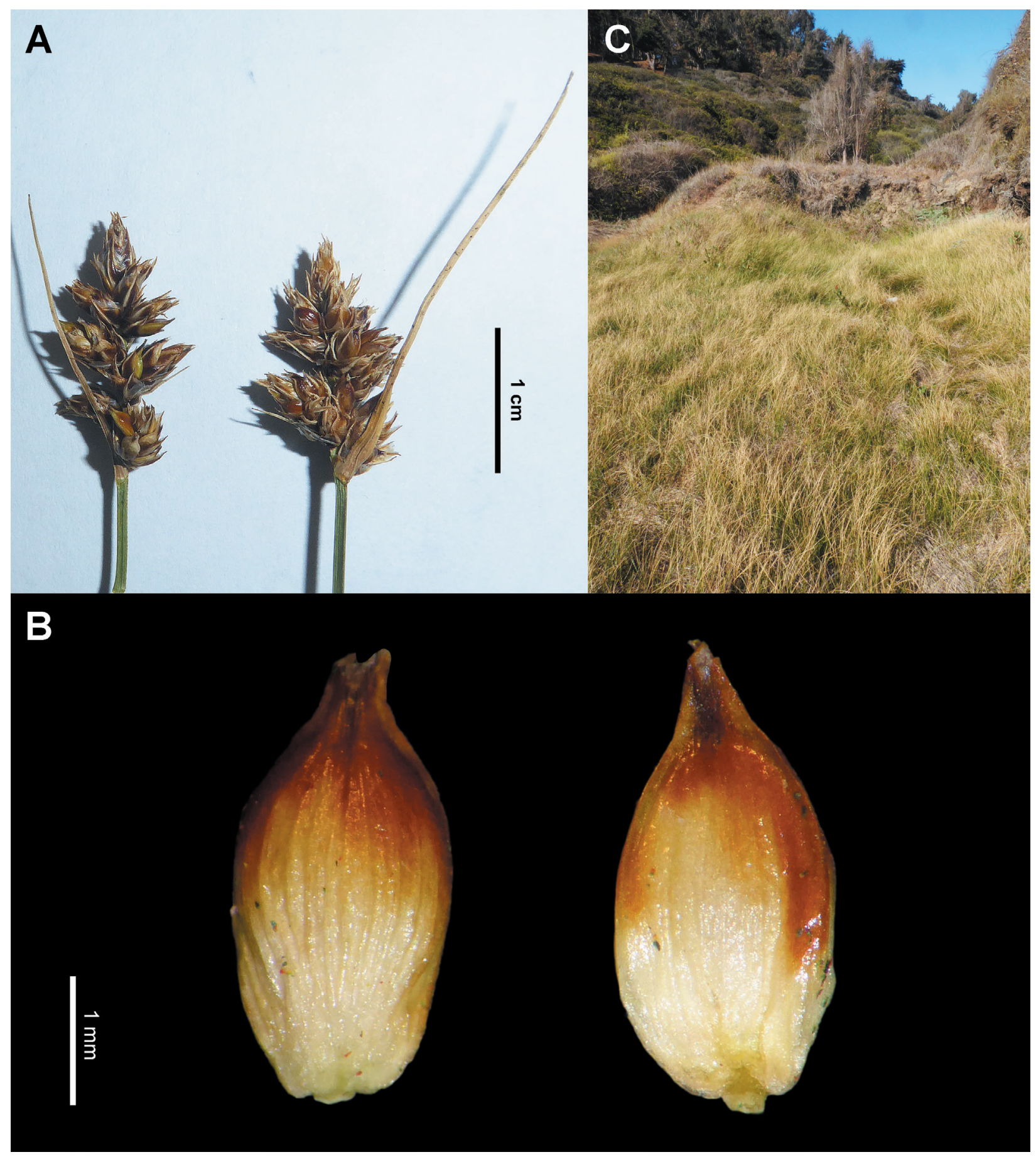

Figura 1. Carex divisa Huds.: (A), espigas; (B), utrículos; (C), hábitat en la localidad de Quintay, Valparaíso, Chile (fotografías: J. Calvo). 
Especimenes: Chile, Valparaíso: Quintay, caleta al sur de playa Chica, matorral nativo y herbazales, $33^{\circ} 12^{\prime} 26^{\prime \prime} \mathrm{S}, 71^{\circ} 41^{\prime} 50^{\prime \prime} \mathrm{W}, 10 \mathrm{~m}, 10 . \mathrm{X} .2019$ (floración), Calvo \& Escobar 8014 (MA, SGO, UPOS); Quintay, caleta al sur de playa Chica, $33^{\circ}$ $12^{\prime} 26^{\prime \prime} \mathrm{S}, 71^{\circ} 41^{\prime} 50^{\prime \prime} \mathrm{W}, 10 \mathrm{~m}$, herbazal, substrato arenoso, 17.XI.2019 (fructificación), Calvo 8035 (CONC, SGO).

\section{AGRADECIMIENTOS}

Agradecemos la contribución de A. Pérez Haase y M. Escudero como revisores, quienes con sus comentarios han contribuido a mejorar una versión previa de esta nota. El primer autor ha sido financiado por FONDECYT a través de una beca postdoctoral (proyecto $\mathrm{n}^{\circ} 3170270$ ); el segundo autor ha sido financiado por la Consejería de Ciencia, Universidades e Innovación de la Comunidad de Madrid, a través del proyecto Macondo (SI1/PJI/2019-00333).

\section{REFERENCIAS BIBLIOGRÁFICAS}

Ball, P. W. \& Reznicek, A. A. 2002. Carex L. In: Flora of North America Editorial Committee (Eds.), Flora of North America North of Mexico 23. Oxford University Press, New York: 254-273.

Barros, M. 1935. Ciperáceas argentinas II, géneros Kyllinga Rottb., Scirpus L., y Carex L. Anales del Museo Argentino de Ciencias Naturales Bernardino Rivadavia 38: 133-264.

Barros, M. 1969. Cyperaceae. In: Correa, M. N. (Ed.), Flora patagónica 2. INTA, Buenos Aires: 38-94.

Egorova, T. V. 1999. The sedges (Carex L.) of Russia and adjacent states. Missouri Botanical Garden Press, Saint Louis.

Govaerts, R., Jiménez-Mejías, P., Koopman, J. et al. 2019. World checklist of Cyperaceae. Royal Botanic Gardens, Kew. Retrieved November 23, 2019, from http://apps. kew.org/wcsp

Herter, W. C. 1953. Flora del Uruguay V, Glumiflorae III. Revista Sudamericana de Botánica 47: 129-173.

Jiménez-Mejías, P., Benítez-Benítez, C., Beltrán, H. et al. 2018a. Carex (Cyperaceae) in South America: diversity, phylogenetics and biogeography of a Boreotemperate element in the Neotropics. Poster presented at the $6^{\text {th }}$ International Conference on Comparative Biology of Monocotyledons. Monocots VI (Natal, Rio Grande do Norte, Brazil, 7-12 October 2018). Retrieved November
25, 2019, from https://www.researchgate.net/publication/329810066_Carex_Cyperaceae_in_South_America_diversity_phylogenetics_and biogeography_of_a_Boreotemperate_element_in the_Neotropics. https://dx.doi. org/10.13140/RG.2.2.15139.30248

Jiménez-Mejías, P., Fabbroni, M., Donadío, S. et al. 2016. Taxonomic and distribution notes on Carex (Cyperaceae) from the Neotropics. Boletin de la Sociedad Argentina de Botánica 51: 727-739. https://doi.org/10.31055/1851.2372. v51.n4.16400

Jiménez-Mejías, P. \& Silva, L. P. 2020. Carex in Flora do Brasil 2020. Jardim Botânico do Rio de Janeiro, Rio de Janeiro. Retrieved November 23, 2019, from http://floradobrasil. jbrj.gov.br/reflora/floradobrasil/FB7161

Jiménez-Mejías, P., Strong, M., Gebauer, S., Hilpold, A., Martín-Bravo, S. \& Reznicek, A. A. 2018b. Taxonomic, nomenclatural and chorological reports on Carex (Cyperaceae) in the Neotropics. Willdenowia 48: 117-124. https:// doi.org/10.3372/wi.48.48108

Kükenthal, G. 1909. Cyperaceae-Caricoideae. In: Engler, A. (Ed.), Das Pflanzenreich. Regni vegetabilis conspectus IV. 20 (Heft 38). Wilhelm Engelmann, Leipzig: 1-824.

Macbride, J. F. 1936. Flora of Peru 1. Field Museum of Natural History, Chicago.

Martín-Bravo, S., Jiménez-Mejías, P., Villaverde, T., Escudero, M., Spalink, D., Roalson, E. H., Hipp, A. L. \& the Global Carex Group. 2019. A tale of worldwide success: behind the scenes of Carex (Cyperaceae) biogeography and diversification. Journal of Systematics and Evolution 57: 695-718. https://doi.org/10.1111/jse.12549

Myndel-Pedersen, T. 1968. Cyperaceae. In: Cabrera, A. L. (Ed.), Flora de la Provincia de Buenos Aires 1. INTA, Buenos Aires: 315-421.

Steyermark, J. A. 1951. Contributions to the Flora of Venezuela. Fieldiana 28: 1-242.

Villaverde, T., González-Moreno, P., Rodríguez-Sánchez, F. \& Escudero, M. 2017. Niche shifts after long-distance dispersal events in bipolar sedges (Carex, Cyperaceae). American Journal of Botany 104: 1765-1774. https://doi. org/10.3732/ajb.1700171

Wheeler, G. A. 1987. The taxonomy of Carex sect. Abditispicae sect. nov. (Cyperaceae) from Austral South America. Systematic Botany 12: 572-585. https://doi. org $/ 10.2307 / 2418892$

Wheeler, G. A. 1989. The taxonomy of Carex sect. Aciculares (Cyperaceae) in South America. Systematic Botany 14: 168-188. https://doi.org/10.2307/2418904

Wheeler, G. A. 1990. Taxonomy of the Carex atropicta complex (Cyperaceae) in South America. Systematic Botany 15: 643-659.

Wheeler, G. A. 2007. Carex and Uncinia (Cyperaceae, Cariceae) from the Juan Fernández Archipelago, Chile. Darwiniana 45: 120-141. https://doi.org/10.2307/2419161 